\title{
Aspectos neuropsiquiátricos da COVID-19
}

\author{
Neuropsychiatric aspects of COVID-19 \\ Aspectos neuropsiquiátricos de COVID-19
}

Recebido: 11/03/2021 | Revisado: 18/03/2021 | Aceito: 21/03/2021 | Publicado: 29/03/2021

\author{
Rafael Lozano de Moraes \\ ORCID: https://orcid.org/0000-0002-6238-2820 \\ Universidade Estadual de Mato Grosso do Sul, Brasil \\ E-mail: rafaellozanodemoraes@gmail.com \\ Thiago Kenzo Nobusa \\ ORCID: https://orcid.org/0000-0002-6461-1279 \\ Universidade Estadual de Mato Grosso do Sul, Brasil \\ E-mail: knobusa@gmail.com \\ José Carlos Souza \\ ORCID: https://orcid.org/0000-0003-4460-3770 \\ Universidade Estadual de Mato Grosso do Sul, Brasil \\ E-mail: josecarlossouza@uol.com.br
}

\begin{abstract}
Resumo
A nova infecção pelo coronavírus, descoberta ao final de 2019, na China, se disseminou pelo mundo rapidamente, atingindo todos os continentes, sendo então declarada uma pandemia pela Organização Mundial de Saúde (OMS). De modo a inibir a transmissão da doença, diversas medidas restritivas foram adotadas por autoridades públicas, o que determinou uma série de alterações no funcionamento da sociedade. Para além da própria doença infecciosa, o COVID-19 tem um profundo impacto na saúde mental da população, com repercussões sociais e neuropsiquiátricas. Sendo assim, o presente trabalho objetivou analisar os principais aspectos envolvidos apontados na literatura, por meio da revisão de estudos realizados com diferentes populações no Brasil e no mundo, delineando assim os principais pontos de discussão. Para cumprir com tal propósito, optou-se por uma revisão bibliográfica do tipo narrativa a partir da busca de artigos referentes aos aspectos social, neuropsiquiátricos e de saúde mental no contexto da pandemia de COVID-19, nas bases de dados "PubMed", "Scielo" e "Google Acadêmico'. Como resultado, observou-se que a pandemia de COVID-19 está envolvida em uma série de alterações na esfera social, com impacto emocional significativo, e repercussões neuropsiquiátricas de modo a causar diversos sintomas associados aos principais transtornos de saúde mental. Nesse sentido, pode-se afirmar, portanto, que a pandemia de COVID-19 deve ser entendida como muito mais que apenas um perigo de doença infecciosa, e que seu impacto na saúde mental da população não deve ser negligenciado, e sim estudado de maneira mais aprofundada e combatido pelas autoridades competentes.
\end{abstract}

Palavras-chave: Coronavírus; Psiquiatria; Neuropsiquiatria; Saúde mental; Depressão; Ansiedade.

\begin{abstract}
The new coronavirus infection, discovered at the end of 2019 in China, spread around the world rapidly, reaching all continents, and was then declared a pandemic by the World Health Organization (WHO). In order to inhibit the transmission of the disease, several restrictive measures were adopted by public authorities, which determined a series of changes in the functioning of society. In addition to the infectious disease itself, COVID-19 has a profound impact on the mental health of the population, with social and neuropsychiatric repercussions. Thus, the present study aimed to analyze the main aspects involved pointed out in the literature, through the review of studies conducted with different populations in Brazil and worldwide, thus outlineing the main points of discussion. To fulfill this purpose, we opted for a narrative literature review based on the search for articles related to social, neuropsychiatric and mental health aspects in the context of the COVID-19 pandemic, in the databases "PubMed", "Scielo" and "Google Scholar". As a result, it was observed that the COVID-19 pandemic is involved in a series of changes in the social sphere, with significant emotional impact, and neuropsychiatric repercussions causing several symptoms associated with major mental health disorders. In this sense, it can be affirmed, therefore, that the COVID-19 pandemic should be understood as much more than just the danger of infectious disease, and that its impact on the mental health of the population should not be neglected, but studied more thoroughly and combated by the competent authorities.
\end{abstract}

Keywords: Coronavirus; Psychiatry; Neuropsychiatry; Mental health; Depression; Anxiety.

\section{Resumen}

La nueva infección por coronavirus, descubierta a finales de 2019 en China, se extendió por todo el mundo rápidamente, llegando a todos los continentes, y luego fue declarada pandemia por la Organización Mundial de la 
Salud (OMS). Con el fin de inhibir la transmisión de la enfermedad, varias medidas restrictivas fueron adoptadas por las autoridades públicas, que determinaron una serie de cambios en el funcionamiento de la sociedad. Además de la enfermedad infecciosa en sí, COVID-19 tiene un profundo impacto en la salud mental de la población, con repercusiones sociales y neuropsiquiátricas. Así, el presente estudio tenía como objetivo analizar los principales aspectos involucrados señalados en la literatura, a través de la revisión de estudios realizados con diferentes poblaciones en Brasil y en todo el mundo, esbozando así los principales puntos de discusión. Para cumplir con este propósito, optamos por una revisión de la literatura narrativa basada en la búsqueda de artículos relacionados con aspectos sociales, neuropsiquiátricos y de salud mental en el contexto de la pandemia COVID-19, en las bases de datos "PubMed", "Scielo" y "Google Scholar". Como resultado, se observó que la pandemia COVID-19 está involucrada en una serie de cambios en la esfera social, con un impacto emocional significativo, y repercusiones neuropsiquiátricas con el fin de causar varios síntomas asociados con los principales trastornos de salud mental. En este sentido, se puede afirmar, por lo tanto, que la pandemia COVID-19 debe entenderse como mucho más que un peligro de enfermedad infecciosa, y que su impacto en la salud mental de la población no debe ser descuidado, sino estudiado más a fondo y combatido por las autoridades competentes.

Palabras clave: Coronavirus; Psiquiatría; Neuropsiquiatría; Salud mental; Depresión; Ansiedad.

\section{Introdução}

Em dezembro de 2019, uma nova mutação viral foi descoberta na cidade de Wuhan (China), dando origem à nova infecção pelo coronavírus, que recebeu o nome de COVID-19. Devido à rápida disseminação do agente, e pelo potencial pandêmico apresentado, a Organização Mundial da Saúde (OMS) declarou, no dia 30 de janeiro de 2020, a COVID-19 como uma emergência de saúde pública internacional, sendo posteriormente, no dia 11 de março de 2020, finalmente considerada como uma pandemia, por ter atingindo países em todos os continentes. De modo a lidar com essa nova situação mundial, diversas medidas restritivas foram adotadas por autoridades governamentais, visando de modo geral reduzir a transmissão do vírus e aliviar o impacto no sistema de saúde público. Assim uma parcela significativa da população mundial, desde então, está restrita aos seus domicílios e, em alguns lugares, também foi instituído o fechamento dos locais não essenciais, chamado lock down. (Dubey et al., 2020).

Em meio a esse contexto de rápida expansão de uma nova doença, associado a uma intensa disseminação de informações, muitas vezes falsas, grande parte da população passou a experienciar altos níveis de problemas emocionais. As próprias medidas de quarentena e distanciamento social adotadas, assim como as repercussões econômicas da pandemia podem ser consideradas causadoras de medo e ansiedade (Miranda et al., 2020). Em uma pandemia, o medo e a insegurança provocam ansiedade e aumento dos níveis de estresse em indivíduos saudáveis, e intensifica os sintomas de indivíduos com transtornos psiquiátricos pré-existentes (Shigemura et al., 2020). Além disso, considera-se que em epidemias, o número de pessoas psicologicamente afetadas costuma superar o número de pessoas acometidas pela infecção (Ornell et al., 2020).

Esse impacto psicoemocional, assim como suas categorias diagnósticas psiquiátricas, tende a ser ainda mais acentuado nos profissionais de saúde ditos da "linha de frente", que atuam diretamente no atendimento aos pacientes portadores de COVID-19 (Hossain et al., 2020). Estes profissionais estão sujeitos a um risco extremamente alto de infecção, e sob contínua pressão relacionada ao trabalho, com aumento na experiência de isolação, risco de adoecimento e longos e estressantes períodos de trabalho. Pesquisas pregressas indicam que em situações de alto risco e estresse em uma epidemia, profissionais da área médica estão mais sujeitos a experenciar uma gama de problemas psicológicos, incluindo medo, depressão, ansiedade, transtorno do estresse pós-traumático e insônia (Liang et al., 2020).

Diante desse novo contexto da pandemia de COVID-19, e considerando seu importante impacto nas esferas biológica, psíquica e social, há de se esperarem numerosas repercussões neuropsiquiátricas na população geral, assim como em trabalhadores de saúde. Frente a esse contexto, considera-se que a abordagem dos aspectos psicopatológicos em uma situação de pandemia não pode ser uma atitude negligenciada. Diante do exposto, o objetivo principal deste estudo é fazer uma discussão sobre os aspectos neuropsiquiátricos da COVID-19, e suas repercussões na saúde mental da população. 


\section{Metodologia}

Trata-se de um estudo de caráter qualitativo e descritivo, através de uma revisão bibliográfica do tipo narrativa. Este tipo de delineamento se torna ideal no contexto de temáticas recentes, uma vez que possibilita a integração e utilização ampliada de fontes de pesquisa, independentemente da sua diversidade de abordagens metodológicas (Crepaldi et al., 2020). Neste contexto, este fato se enquadra na questão da pandemia pelo COVID-19, uma situação emergente com surgimento constante de novos conhecimentos que, por conseguinte, requerem igual eficácia e ritmo no âmbito das produções científicas (Schmidt et al., 2020).

No desenvolver deste estudo, foram selecionados artigos científicos a partir dos bancos de dados bibliográficos Scientific Eletronic Library Online (SciELO), National Library of Medicine (PubMed) e "Google Acadêmico", sem se restringir ao idioma português e buscando publicações dos anos 2019 a 2021. A busca foi realizada nos meses de janeiro e fevereiro de 2021, a partir do uso dos seguintes descritores e palavras-chaves, acrescidos dos booleanos "e" e "AND", para refinamento da pesquisa: "Coronavírus"; "psiquiatria"; "neuropsiquiatria"; "saúde mental"; "pandemia"; "epidemias"; “intervenção na crise"; "surtos de doenças"; “depressão"; “ansiedade”; "sono”; “infecções por Coronavírus”; “inquéritos epidemiológicos" e "Classificação Internacional de Doenças". Após o levantamento dos artigos, por meio da leitura do título e do resumo, foram selecionados aqueles que apresentavam pertinência ao tema "aspectos neuropsiquiátricos da COVID-19", caso contrário, eram descartados.

Após análise da bibliografia, foi delimitada uma linha de raciocínio narrativo e de trabalho que se desenvolveria no estudo, culminando na determinação de dois tópicos, de forma a apresentar os dados de maneira didática, sendo eles: (1) a COVID-19 e a repercussão social e (2) repercussões neuropsiquiátricas da COVID-19.

\section{Resultados e Discussão}

\subsection{A COVID-19 e a repercussão social}

Ao longo da sua história, a humanidade passou por diversos cenários de doenças infecciosas que se constituíram em epidemias, causando grandes impactos e pressões negativas sobre as sociedades atingidas, tais como herpes, legionelose, AIDS, Ebola e Síndrome Respiratória Aguda Grave (SARS) e, atualmente, a COVID-19, a qual, com seu caráter altamente contagioso, alcançou proporções globais, dando início a uma pandemia com indiscutíveis efeitos na saúde internacional. Diferentemente de outras doenças, aquelas que possuem potencialidade pandêmica têm sua gravidade alojada para além da simples ação do patógeno no sistema biológico do indivíduo, uma vez que revela as condições sociais e põe à prova as sociedades atingidas, através da imposição de novos cenários de enfrentamento, sendo estes igualmente ou ainda piores que a própria doença (Jones et al., 2020).

Os meios de comunicação social e a mídia em geral detêm grande poder nesse contexto; enquanto podem garantir rápida disseminação de informações com influência suficiente para determinar padrões sociais, como continuidade ou suspensão de determinados serviços e restrições de tráfego, seja positiva ou negativamente (Depoux et al., 2020), ao mesmo tempo podem representar ameaças no controle da pandemia. A chamada “infodemia do coronavírus" é um evento que ocorre promovendo informações exageradas, rumores e sensacionalismo sobre a doença, através principalmente das redes sociais, onde a COVID-19 se torna o assunto popular, levando grupos ou pessoas a disseminar informações alteradas e gerando ainda mais terror. Ao mesmo tempo, também existem indivíduos que compartilham e forjam informações e conteúdos que diminuem a importância e a gravidade da pandemia; isto influencia na percepção diminuída e errônea sobre a mesma (Dubey et al., 2020).

O mau uso das redes sociais e informacionais também desempenha papel dentro da formação e fortalecimento de estigmas e preconceitos em relação à COVID-19. Com a ascensão da doença, é presumível que seja criados fatores como medo 
do isolamento, racismo, discriminação e marginalização, além das suas consequências nos campos sociais e econômicos; o impacto disso se reflete em populações que não procuram atendimento no tempo devido, omitindo informações importantes para o raciocínio profissional no caso do indivíduo e para a saúde coletiva (Dubey et al., 2020). Outro estigma é do sentimento xenofóbico em relação aos chineses como responsáveis pelo vírus, o qual ainda é ampla e erroneamente divulgado, além de configurar ato de racismo. Esse sentimento também é apontado com o cuidado para que não constitua fator de influência negativa na tomada de medidas de saúde pública, ao direcionar o ódio e preconceito a um grupo específico (Chung et al., 2020).

As pandemias podem ser caracterizadas por uma perda massiva, seja ela de rotinas, vínculos afetivos presenciais, qualidade de vida financeira e mesmo vidas de desconhecidos e conhecidos, inserindo-se, neste último, o processo do luto. Ao perder um ente querido, passa-se por processo doloroso, o qual é atenuado pelos "rituais de despedida". Os rituais são momentos marcados pela lembrança e reflexão de bons momentos, questões não resolvidas, perdões e agradecimentos, em vista a facilitar a resolução do luto. Entretanto, de forma singular a outros eventos com grande quantidade de mortes, o caso da COVID-19 não permitiu ou limitou em muito a execução desses momentos, fato que repercute diretamente no bem-estar mental daqueles afetados pela perda, que, sem poder despedir-se daqueles que partiram, ainda deve passar pelo processo de perda de forma mais solitária, ao passo em que não pode contar com grande apoio emocional das várias pessoas presentes nesses momentos e que compartilham o luto. Há outros fatores que alteram a forma comum pela qual o luto era enfrentado socialmente fora da pandemia, sendo um deles os casos em que há múltiplas perdas, situação não tão rara, considerando que a transmissibilidade da doença está ligada ao contato próximo entre os indivíduos (Crepaldi et al., 2020). Ligado ao término da vida, também houve alterações nos processos anteriores à morte, como na impossibilidade de contato com paciente, uma vez que ele seja internado, o que torna a situação ainda mais difícil pela solidão ou sentimento de não estar junto, sendo permitido somente o contato através do uso de aparelhos eletrônicos (como celulares), quando permitidos (Ingravallo et al., 2020).

Dentro dos determinantes do processo saúde-doença sociais, a quarentena tem grande impacto. Ao mesmo tempo que, se utilizada adequadamente, constitui meio para combater a COVID-19, ela também apresenta repercussões negativas, especialmente no campo psicossocial (Brooks et al., 2020), resultando no prejuízo da saúde mental da população (Gualano et al., 2020). As consequências sociais observadas pela quarentena que se instituiu com a COVID-19 incluem a separação de familiares, isolamento social, insuficiência no fornecimento de itens essenciais, perdas econômicas, entre outros, que contribuem na geração de histeria, ansiedade, estresse e sofrimento (Dubey et al., 2020). Com a suspensão de setores da economia e colapso de alguns deles, pode-se desencadear efeitos e prejuízos tanto de longo prazo dentro da sociedade, levando anos para haver recuperação (Rubin et al., 2020), quanto aos próprios indivíduos com a danos financeiros e risco de perder o emprego, experiências de grande carga negativa (Ho et al., 2020).

Apesar da COVID-19 ter causado mudanças na estrutura social que são inerentes à maior parte da população, grupos específicos também tiveram suas peculiaridades, como é o caso dos profissionais de saúde (principalmente da linha de frente contra a pandemia), crianças, idosos, comunidade marginalizada (migrantes, moradores de favelas, presidiários), entre outros (Dubey et al., 2020).

A necessidade por profissionais de saúde, em momento de emergência, fez com que vários cuidadores ficassem sobrecarregados; isto veio a desencadear um estresse ocupacional relacionado ao trabalho (Burnout), presenteísmo (trabalhar doente) e absenteísmo (ficar afastado do trabalho por motivo de doença). Há grande pressão por se tratar de uma doença emergente e desconhecida em vários locais e países que não contavam com estrutura adequada para receber ou tratar da grande demanda da COVID-19. Os recursos escassos, falha no cuidado do paciente, mortes constantes, sentimento de incapacidade, proteção inadequada contra a contaminação e mortes de colegas de trabalho foram algumas das adversidades que esse grupo teve que enfrentar, que juntamente à carga trabalho e impossibilidade de ver a família, principalmente pelo risco de transmitir a 
doença, contribuíram e contribuem no adoecimento mental dos profissionais de saúde (Dubey et al., 2020).

Com o fechamento de escolas e espaços de lazer, bem como a impossibilidade de contato com pessoas de fora da residência, as crianças ficaram confinadas apenas ao ambiente familiar da própria casa (Dubey et al., 2020). Essa mudança repentina no ambiente e nas relações diárias, juntamente à quarentena com os pais, muitas vezes já também estressados por vários outros motivos inerentes à pandemia, causa grande estresse às crianças. No entanto, o quadro torna-se ainda mais grave quando a criança é separada de seus pais ou cuidadores, por conta de estarem na linha de frente contra a COVID-19, doentes, a própria criança estar doente (requirindo o isolamento desta) ou mesmo terem ido a óbito por conta da pandemia. Estes constituem problemas sérios no desenvolvimento psicológico e, posteriormente, influenciando na inserção social do indivíduo, podendo o trauma desenvolver repercussões a longo prazo como maiores riscos de desenvolver desordens de humor, psicoses e também levar ao suicídio (Lui et al., 2020). Em casos mais leves, mas ainda prejudiciais ao desenvolvimento emocional e social das crianças, residem nos traumas mais leves, mas em que há percepção de situações infelizes decorrentes da pandemia, como perda de emprego dos pais, morte de familiares e conhecidos, vulnerabilidade e risco dos avós e a perda da segurança depositada em determinados locais e situações por conta do vírus, como, por exemplo, nas escolas (Weaver et al., 2020).

Por conta das vulnerabilidades da população idosa contra a COVID-19 e o constante reforço dessa ideia, esse grupo populacional pode criar considerável medo constante e pavor de ser infectado (Dubey et al., 2020). Os idoso que apresentam declínio cognitivo ou demências, podem estar mais propensos a alterações de comportamento e necessitam de melhores informações e esclarecimentos sobre a pandemia, sua gravidade e os cuidados necessários. Dentro deste cenário, há várias iniciativas de apoio a esses indivíduos, sobretudo quando residem sozinhos, como em necessidades para compras e outras situações em que seria necessária a saída da moradia (Rubin et al., 2020).

As comunidades marginalizadas, como migrantes e residentes de favelas, encontram-se em situações de infraestrutura pública de saúde precária, bem como menores condições para higiene e ambientes com adequado saneamento, fatores esses que não são favoráveis ao cenário pandêmico da COVID-19. Da mesma forma, muitos vivem de forma aglomerada constantemente por não possuir estrutura e espaço na moradia, aumentando o risco ao contágio da doença. Há ainda a discriminação e xenofobia no caso dos migrantes, os quais são julgados e condenados pela população local como responsáveis pela chegada e disseminação do vírus na região (Dubey et al., 2020). No caso do sistema prisional, é considerada a saúde prisional como saúde pública. Isso se dá ao fato de prisões constituírem epicentros para a doenças como a COVID-19, com pouco espaço para a prática adequada de isolamento (muitas vezes havendo superlotação), compartilhamento de espaços comuns e higiene prejudicada por políticas que estabelecem limites ao acesso de sabão e álcool, se tornam locais extremamente favoráveis à disseminação, uma vez que o agente tenha entrado por meio do fluxo constante de agentes penitenciários, trabalhadores, visitantes e presos (Carvalho et al., 2020).

Outro grupo social de preocupação seriam os "sem-tetos", os quais, não possuindo condições para realizar isolamento, higiene adequada ou manter distanciamento social, estão extremamente vulneráveis à doença (Dubey et al., 2020).

Apesar da COVID-19 se caracterizar como uma doença infecto contagiosa com manifestações semelhantes ao estado gripal (Xavier et al., 2020), não se deve restringir apenas ao tratar da doença, devendo, o estado pandêmico, ser entendido de forma ampla como um grande preditor de repercussões sociais que, como uma das suas produções, apresenta diversas manifestações psicossociais.

\subsection{Repercussões neuropsiquiátricas da COVID-19}

A nova infecção pelo coronavírus, ou COVID-19, se tornou uma pandemia de importante impacto na saúde mundial, afetando as esferas biológica, social e psicológica da população como um todo. Tanto as pessoas acometidas pelo COVID-19, assim como aquelas vivenciando os efeitos das medidas restritivas adotadas no período de pandemia, estão mais sujeitas à 
problemas de saúde mental, incluindo depressão, ansiedade, estresse, ataques de pânico, raiva irracional, impulsividade, transtorno de somatização, distúrbios do sono, transtorno do estresse pós-traumático e comportamento suicida (Hossain et al., 2020). O estresse agudo e sintomas ansiosos e depressivos vivenciados por esses indivíduos podem evoluir para ataques de pânico, transtornos de ansiedade, transtorno do estresse pós-traumático, depressão e até suicídio (Shigemura et al., 2020).

Os transtornos mentais mais prevalentes na população durante a pandemia de COVID-19, assim como seus principais impactos nas esferas física, emocional e social, definidos com base na Classificação de Transtornos mentais e de Comportamento da Classificação Internacional de Doenças, versão número 10, da Organização Mundial de Saúde - CID-10, podem ser visualizados no Quadro 1. 
Quadro 1 - Transtornos mentais e seus principais comprometimentos durante a pandemia da COVID-19.

\begin{tabular}{|c|c|c|c|}
\hline Comprometi & Físico & Emocional & Social \\
\hline $\begin{array}{l}\text { Episódio } \\
\text { depressivo }\end{array}$ & $\begin{array}{c}\text { Insônia, hipobulia e } \\
\text { anergia }\end{array}$ & $\begin{array}{l}\text { Hipotimia, anedonia, } \\
\text { irritabilidade }\end{array}$ & $\begin{array}{c}\text { Isolamento, descrença } \\
\text { na família e amigos }\end{array}$ \\
\hline $\begin{array}{l}\text { Transtorno de } \\
\text { ansiedade } \\
\text { generalizada }\end{array}$ & $\begin{array}{l}\text { Hiperatividade, } \\
\text { insônia e inquietude }\end{array}$ & $\begin{array}{c}\text { Nervosismo, } \\
\text { preocupação, } \\
\text { apreensão e medo }\end{array}$ & $\begin{array}{c}\text { Isolamento e } \\
\text { afastamento de } \\
\text { pessoas próximas }\end{array}$ \\
\hline $\begin{array}{l}\text { Transtorno } \\
\text { Obsessivo- } \\
\text { compulsivo }\end{array}$ & $\begin{array}{l}\text { Comportamento } \\
\text { estereotipado e } \\
\text { obsessões }\end{array}$ & $\begin{array}{l}\text { Angústia, frustração e } \\
\text { compulsividade }\end{array}$ & $\begin{array}{c}\text { Alterações de conduta } \\
\text { e personalidade }\end{array}$ \\
\hline $\begin{array}{l}\text { Transtorno do } \\
\text { pânico }\end{array}$ & $\begin{array}{l}\text { Episódios graves de } \\
\text { hiperatividade } \\
\text { autonômica }\end{array}$ & $\begin{array}{l}\text { Apreensão, medo e } \\
\text { ansiedade }\end{array}$ & $\begin{array}{c}\text { Evitação de } \\
\text { ambientes e situações } \\
\text { sociais }\end{array}$ \\
\hline $\begin{array}{l}\text { Transtorno do } \\
\text { estresse pós- } \\
\text { traumático }\end{array}$ & $\begin{array}{l}\text { Hipervigilância, } \\
\text { agressividade e } \\
\text { insônia }\end{array}$ & $\begin{array}{c}\text { Embotamento } \\
\text { emocional, anedonia }\end{array}$ & Isolamento, evitação \\
\hline $\begin{array}{c}\text { Fobia social e } \\
\text { específica }\end{array}$ & $\begin{array}{l}\text { Episódios ansiosos } \\
\text { provocados por } \\
\text { objeto/situação }\end{array}$ & $\begin{array}{l}\text { Medo, ansiedade e } \\
\text { preocupação }\end{array}$ & $\begin{array}{c}\text { Evitação de situações } \\
\text { sociais e específicas }\end{array}$ \\
\hline Agorafobia & $\begin{array}{c}\text { Incapacitação } \\
\text { associada a medo } \\
\text { intenso }\end{array}$ & $\begin{array}{l}\text { Ansiedade e medo } \\
\text { associados a situações } \\
\text { específicas }\end{array}$ & $\begin{array}{c}\text { Evitação de } \\
\text { aglomerações e } \\
\text { espaços públicos }\end{array}$ \\
\hline $\begin{array}{l}\text { Dependência de } \\
\text { álcool }\end{array}$ & $\begin{array}{l}\text { Desejo pelo álcool, } \\
\text { abstinência e prejuízo } \\
\text { cognitivo }\end{array}$ & $\begin{array}{l}\text { Irritabilidade, abandono } \\
\text { de interesses cotidianos }\end{array}$ & $\begin{array}{l}\text { Deterioração das } \\
\text { relações sociais pela } \\
\text { adicção }\end{array}$ \\
\hline $\begin{array}{l}\text { Dependência de } \\
\text { drogas lícitas }\end{array}$ & $\begin{array}{l}\text { Desejo pela droga, } \\
\text { abstinência e prejuízo } \\
\text { cognitivo }\end{array}$ & $\begin{array}{l}\text { Irritabilidade, abandono } \\
\text { de interesses cotidianos }\end{array}$ & $\begin{array}{l}\text { Deterioração das } \\
\text { relações sociais pela } \\
\text { adicção }\end{array}$ \\
\hline $\begin{array}{l}\text { Dependência de } \\
\text { drogas ilícitas }\end{array}$ & $\begin{array}{l}\text { Desejo pela droga, } \\
\text { abstinência e prejuízo } \\
\text { cognitivo }\end{array}$ & $\begin{array}{l}\text { Irritabilidade, } \\
\text { anedonia e } \\
\text { instabilidade }\end{array}$ & $\begin{array}{c}\text { Alienação social e } \\
\text { comportamento de } \\
\text { risco }\end{array}$ \\
\hline
\end{tabular}

Fonte: Autores (2021).

Estudos realizados com população geral na China evidenciaram que as prevalências dos principais transtornos de saúde mental, associados à sua sintomatologia e principais repercussões em diferentes esferas, identificados no Quadro 1, sofreram um aumento quando comparadas ao período anterior à pandemia, evidenciando o importante impacto do COVID-19 
na saúde mental da população geral (Shi et al., 2020). O isolamento social e a experiência de medo e ansiedade percebidos por vários indivíduos, em especial os portadores de transtornos de saúde mental, impulsionaram um grande número de pessoas ao uso de substâncias lícitas e ilícitas como uma forma de estratégia de enfrentamento, o que aumenta o risco de desenvolvimento de dependência física e psicológica por essas substâncias (Zaami, Marinelli, \& Vari, 2020).

O transtorno do estresse pós-traumático (TEPT) é um transtorno comum, grave e complexo que ocorre após exposição a eventos traumáticos. É caracterizado por invasão de pensamentos e constante reexperienciamento do trauma vivenciado por meio de pesadelos e flashbacks, com extensa gama de sintomas associados, como embotamento emocional, anedonia, hipervigilância e insônia. Estudos populacionais realizados na China revelaram um aumento na prevalência desse transtorno no período de pandemia, especialmente em grupos considerados vulneráveis, como pacientes positivos para COVID-19, profissionais de saúde de linha de frente e indivíduos em quarentena, representando um grave problema de saúde pública mental a ser enfrentado (Yuan et al., 2021).

Um dos pilares da saúde mental que foi afetado de maneira significativa pela pandemia de COVID-19 foi a manutenção adequada do sono, predispondo os indivíduos à vários distúrbios do sono e, sendo um dos mais importantes a insônia. A insônia é um sério problema de saúde, com grande impacto psicológico, e associado a uma piora do desempenho físico e mental durante o dia. Altos níveis de intolerância às incertezas, preocupações relacionadas ao COVID-19, solidão e sintomas depressivos são preditores de insônia (Voitsidis et al., 2020). Um estudo chinês realizado com a população geral demonstrou um aumento significativo na prevalência de insônia, com surgimento de novos casos e piora de sintomas de insônia pré-existentes, assim como aumento do período de latência do sono, associados a sintomas de depressão e ansiedade, especialmente em indivíduos do sexo feminino e portadores de transtornos psiquiátricos (Li et al., 2020). Outra investigação, realizada com população geral francesa, confirmou que preocupações relacionadas ao COVID-19 e solidão associada ao isolamento social são fatores decisivos para o desenvolvimento de insônia clínica, assim como menores níveis educacionais, infecção pelo coronavírus e condições psiquiátricas pré-existentes (Kokou-Kpolou et al., 2020). Em profissionais de saúde, uma revisão sistemática revelou que até quatro a cada dez trabalhadores chegaram a experenciar dificuldade de dormir e/ou insônia, muitas vezes em associação com sintomas ansiosos e depressivos (Pappa et al., 2020).

A distribuição epidemiológica de problemas de saúde mental e fatores associados se manifestaram de maneira heterogênea entre o público geral, pacientes com COVID-19 e profissionais de saúde (Hossain et al., 2020). Muitos fatores psicossociais, como membros da família sendo infectados, exposição massiva à mídia e redes sociais, precário apoio social, idade avançada e baixo nível socioeconômico, estão associados a um risco aumentado de prejuízos à saúde mental (Ma et al., 2020). Pacientes portadores de condições neuropsiquiátricas pré-existentes apresentam um risco considerável de experienciar um declínio significativo em sua saúde física e mental, com piora da sintomatologia atual (Dubey et al., 2020). Outro grupo considerado como de maior risco engloba os profissionais de saúde, especialmente aqueles envolvidos diretamente com os cuidados aos portadores de COVID-19. A exposição constante aos casos da doença e um aumento da percepção do risco representado pelo coronavírus, somados ao estresse da rotina de trabalho e uma vivência de maior isolamento social, são fatores que impactam negativamente o bem-estar mental desses indivíduos (Dubey et al., 2020).

Estudos empíricos indicam que pacientes que testaram positivo para COVID-19 apresentaram piora no estado de saúde mental, com elevação nos níveis de depressão, ansiedade e transtorno do estresse pós-traumático, além de deterioração da qualidade do sono, quando comparados a indivíduos controle negativos para COVID (Hossain et al., 2020). Comparados com indivíduos não infectados pelo coronavírus, pacientes com COVID-19 se apresentaram duas a três vezes mais propensos a relatar sintomas de saúde mental, sendo os principais depressão, ansiedade, insônia e estresse; familiares e amigos desses pacientes também experienciaram um aumento na apresentação desses sintomas (Shi et al., 2020).

Com base na nos estudos observados, evidencia-se que a pandemia de COVID-19 trouxe uma série de repercussões 
neuropsiquiátricas de grande relevância para a saúde pública mental da população, acometendo uma parcela significativa de indivíduos, especialmente os considerados de maior risco, como portadores de transtornos mentais, pacientes positivos para COVID-19, profissionais de saúde de linha de frente e indivíduos com redes precárias de apoio social (Hossain et al., 2020). O aumento considerável na prevalência de sintomas de saúde mental, como depressão, ansiedade e insônia, assim como transtornos mentais propriamente definidos, representa um grande desafio a ser enfrentado pelo sistema de saúde pública de diversos países, exigindo intervenções psicossociais complexas e abrangentes de maneira a zelar pela saúde mental da população em um período de calamidade mundial sem precedentes.

\section{Conclusão}

Apesar do agente SARS-CoV-2 caracterizar uma doença infectocontagiosa, cujas principais repercussões fisiopatológicas se refletem no sistema respiratório do indivíduo, fica claro que o episódio da pandemia pela COVID-19 não se limitou apenas a este âmbito, apresentando desafios e problemáticas para as mais diversas áreas e, dentre elas, uma que está presente de forma pronunciada é o impacto neuropsiquiátrico na população.

Não só pelo constante medo e preocupação com as altas taxas de mortalidade e transmissibilidade da doença, mas também por todas as condições impostas pela pandemia como, quarentena, isolamento social, suspensão de atividades econômicas e de lazer, confinamento domiciliar, dentre tantas outras, que se pode afirmar que o evento da COVID-19 é fator de grande nocividade à saúde e bem-estar mentais dos indivíduos. Dentro deste contexto, constata-se que houve importantes mudanças no panorama social, alguns dos quais sendo comuns à maior parte da população (como o estigma da doença, o sentimento xenofóbico contra às pessoas chinesas, as alterações no processo de despedida e do luto, prejuízos financeiros ou na carreira profissional e adversidades familiares), mas também atingindo de forma específica e com maior gravidade determinados grupos sociais (como profissionais de saúde, crianças, idosos e populações marginalizadas).

Com todas essas alterações em suas vidas, somadas ao medo e à insegurança constantes pela doença, muitas vezes agravados com o conflito entre informações verdadeiras e falsas (amplamente disseminadas pelos meios digitais), resultou em um aumento expressivo na prevalência de sintomas de natureza mental, como depressão, ansiedade, insônia e estresse, bem como dos próprios transtornos psiquiátricos, como é o caso de transtornos de ansiedade generalizada, obsessivo-compulsivo, do pânico, do estresse pós-traumático e vários outros problemas mentais e do comportamento, especialmente naqueles indivíduos atingidos mais diretamente, como ao positivar para a doença ou ter alguém próximo que foi infectado.

Visto que ainda há muito a se aprender sobre a nova patologia, é de igual importância que haja continuidade e aprofundamento dos estudos e discussões sobre as suas repercussões no processo saúde-doença, ao considerar a COVID-19 como um evento global, o qual trouxe consigo incontáveis modificações na sociedade, e que, por sua vez, possuem todo o potencial para impactar e pôr em risco o bem-estar e a saúde mental de toda a população.

Por se tratar de um processo ainda em progressão e com suas repercussões pouco conhecidas, torna-se imprescindível a realização de novos estudos de caráter observacional em suas diversas modalidades, destacando-se a possibilidade para a descrição e a análise dos efeitos da pandemia na saúde mental, ao comparar as peculiaridades dos impactos nos diferentes grupos sociais e realizando investigações com abordagem longitudinal, acompanhando assim, as futuras repercussões e manifestações psiquiátricas.

\section{Referências}

Brooks, S. K., Webster, R. K., Smith, L. E., Woodland, L., Wessely, S., Greenberg, N., \& Rubin, G. J. (2020). The psychological impact of quarantine and how to reduce it: rapid review of the evidence. The Lancet, 395(10227), 912-920. https://doi.org/10.1016/s0140-6736(20)30460-8

Carvalho, S. G., Santos, A. B. S., \& Santos, I. M. (2020). A pandemia no cárcere: intervenções no superisolamento. Ciência \& Saúde Coletiva, 25(9), 34933502. https://doi.org/10.1590/1413-81232020259.15682020 
Chung, R. Y., e Li, M. M. (2020). Sentimento anti-chinês durante o surto de nCoV de 2019. Lancet (Londres, Inglaterra), 395(10225), 686687. https://doi.org/10.1016/S0140-6736(20)30358-5

Crepaldi, M. A., Schmidt, B., Noal, D. S., Bolze, S. D. A., \& Gabarra, L. M. (2020). Terminalidade, morte e luto na pandemia de COVID-19: demandas psicológicas emergentes e implicações práticas. Estudos de psicologia (Campinas), 37, e200090, 1982-0275. https://doi.org/10.1590/1982$0275202037 \mathrm{e} 200090$

Depoux, A., Martin, S., Karafillakis, E., Preet, R., Wilder-Smith, A., \& Larson, H. (2020). The pandemic of social media panic travels faster than the COVID19 outbreak. Journal of travel medicine, 27(3), 031. https://doi.org/10.1093/jtm/taaa031

Dubey, S., Biswas, P., Ghosh, R., Chatterjee, S., Dubey, M. J., Chatterjee, S., Lahiri, D., \& Lavie, C. J. (2020). Psychosocial impact of COVID-19. Diabetes \& metabolic syndrome, 14(5), 779-788. https://doi.org/10.1016/j.dsx.2020.05.035

Gualano, M. R., Lo Moro, G., Voglino, G., Bert, F., \& Siliquini, R. (2020). Effects of Covid-19 Lockdown on Mental Health and Sleep Disturbances in Italy. International Journal of Environmental Research and Public Health, 17(13), 4779. https://doi.org/10.3390/ijerph17134779

Ho, C. S., Chee, C. Y., \& Ho, R. C. (2020). Mental Health Strategies to Combat the Psychological Impact of COVID-19 Beyond Paranoia and Panic. Annals of the Academy of Medicine, Singapore, 49(3), 155-160

Hossain, $\quad$ M. M., Tasnim, $\quad$ S., $\quad$ Sultana, $\quad$ A., Faizah, $\quad$ F., Mazumder, $\quad$ H., Zou, $\quad$ L., McKyer, $\quad$ E., $\quad$ Ahmed, $\quad$ H. $\quad$ U., $\quad$ \& Ma, $\quad$ P. (2020). Epidemiology of mental health problems in COVID-19: a review. F1000Research, 9, 636. https://doi.org/10.12688/f1000research.24457.1

Ingravallo, F. (2020). Death in the era of the COVID-19 pandemic. The Lancet Public Health, 5(5), e258. https://doi.org/10.1016/s2468-2667(20)30079-7

Jones, D. S. (2020). History in a Crisis - Lessons for Covid-19. New England Journal of Medicine, 382(18), 1681-1683. https://doi.org/10.1056/nejmp2004361

Kokou-Kpolou, C. K., Megalakaki, O., Laimou, D., \& Kousouri, M. (2020). Insomnia during COVID-19 pandemic and lockdown: Prevalence, sever ity, and associated risk factors in French population. Psychiatry research, 290, 113128. https://doi.org/10.1016/j.psychres.2020.113128

Li, Y., Qin, Q., Sun, Q., Sanford, L. D., Vgontzas, A. N., \& Tang, X. (2020). Insomnia and psychological reactions during the COVID-19 outbreak in China. Journal of Clinical Sleep Medicine, 16(8), 1417-1418. https://doi.org/10.5664/jcsm.8524

Liang, Y., Wu, K., Zhou, Y., Huang, X., Zhou, Y., \& Liu, Z. (2020). Mental Health in Frontline Medical Workers during the 2019 Novel Coronavirus Disease Epidemic in China: A Comparison with the General Population. International Journal of Environmental Research and Public Health, 17(18), 6550. 10.3390/ijerph17186550

Liu, J. J., Bao, Y., Huang, X., Shi, J., \& Lu, L. (2020). Mental health considerations for children quarantined because of COVID-19. The Lancet Child \& Adolescent Health, 4(5), 347-349. https://doi.org/10.1016/s2352-4642(20)30096-1

Ma, Z., Zhao, J., Li, Y., Chen, D., Wang, T., Zhang, Z., Chen, Z., Yu, Q., Jiang, J., Fan, F., \& Liu, X. (2020). Mental health problems and correlates among 746217 college students during the coronavirus disease 2019 outbreak in China. Epidemiology and psychiatric sciences, 29, e181. https://doi.org/10.1017/S2045796020000931

MirandaT. S., SoaresG. F. G., AraujoB. E., FagundesG. H. A., do AmaralH. L. P., SoaresH. C., TavaresK. S., de FassioL. R., MotaT. do N., \& GonçalvesY. de A. (2020). Incidência dos casos de transtornos mentais durante a pandemia da COVID-19. Revista Eletrônica Acervo Científico, 17, e4873. https://doi.org/10.25248/reac.e4873.2020

Ornell, F., Schuch, J. B., Sordi, A. O., \& Kessler, F. H. P. (2020). "Pandemic fear" and COVID-19: mental health burden and strategies. Brazilian Journal of Psychiatry, 42(3), 232-235. https://doi.org/10.1590/1516-4446-2020-0008

Pappa, S., Ntella, V., Giannakas, T., Giannakoulis, V. G., Papoutsi, $\quad$ E., $\quad$ \& Katsaounou, $\quad$ P. (2020). Prevalence of depression, anxiety, and insomnia among healthcare workers during the COVID-19 pandemic: A systematic review and meta-analysis. Brain, Behavior, and Immunity, 88, 901907. https://doi.org/10.1016/j.bbi.2020.05.026

Rubin, G. J., \& Wessely, S. (2020b). The psychological effects of quarantining a city. BMJ, m313. https://doi.org/10.1136/bmj.m313

Shi, L., Lu, Z. A., Que, J. Y., Huang, X. L., Liu, L., Ran, M. S., Gong, Y. M., Yuan, K., Yan, W., Sun, Y. K., Shi, J., Bao, Y. P., \& Lu, L. (2020). Prevalence of and Risk Factors Associated With Mental Health Symptoms Among the General Population in Disease 2019 Pandemic. JAMA network open, 3(7), e2014053. https://doi.org/10.1001/jamanetworkopen.2020.14053

Shigemura, J., Ursano, R. J., Morganstein, J. C., Kurosawa, M. and Benedek, D. M. (2020), Public responses to the novel 2019 coronavirus (2019-nCoV) in Japan: Mental health consequences and target populations. Psychiatry Clin. Neurosci, 74: 281-282. https://doi.org/10.1111/pcn.12988

Voitsidis, P., Gliatas, I., Bairachtari, V., Papadopoulou, K., Papageorgiou, G., Parlapani, E., Syngelakis, M., Holeva, V., \& Diakogiannis, I. (2020). Insomnia during the COVID-19 pandemic in a Greek population. Psychiatry Research, 289, 113076. https://doi.org/10.1016/j.psychres.2020.113076

Weaver, M. S., \& Wiener, L. (2020). Applying Palliative Care Principles to Communicate With Children About COVID-19. Journal of Pain and Symptom Management, 60(1), e8-e11. https://doi.org/10.1016/j.jpainsymman.2020.03.020

Xavier, A. R., Silva, J. S., Almeida, J. P. C. L., Conceição, J. F. F., Lacerda, G. S., \& Kanaan, S. (2020). COVID-19: manifestações clínicas e laboratoriais na infecção pelo novo coronavírus. Jornal Brasileiro de Patologia e Medicina Laboratorial, 56, e3232020. Epub July 01, 2020. https://doi.org/10.5935/16762444.20200049

Yuan, K., Gong, Y. M., Liu, L., Sun, Y. K., Tian, S. S., Wang, Y. J., Zhong, Y., Zhang, A. Y., Su, S. Z., Liu, X. X., Zhang, Y. X., Lin, X., Shi, L., Yan, W., Fazel, S., Vitiello, M. V., Bryant, R. A., Zhou, X. Y., Ran, M. S., Bao, Y. P., \& Lu, L. (2021). Prevalence of posttraumatic stress disorder 
Research, Society and Development, v. 10, n. 4, e3210413803, 2021

(CC BY 4.0) | ISSN 2525-3409 | DOI: http://dx.doi.org/10.33448/rsd-v10i4.13803

after infectious disease pandemics in the twenty-first century, including COVID-19: a meta-analysis and systematic review. Molecular psychiatry, 1-17. https://doi.org/10.1038/s41380-021-01036-x.

Zaami, S., Marinelli, E., \& Varì, M. R. (2020b). New Trends of Substance Abuse During COVID-19 Pandemic: An International Perspective. Frontiers in Psychiatry, 11, 700. https://doi.org/10.3389/fpsyt.2020.00700. 